\title{
Stability Analysis and Stabilization of T-S Fuzzy Delta Operator Systems with Time-Varying Delay via an Input-Output Approach
}

\author{
Zhixiong Zhong, ${ }^{1}$ Guanghui Sun, ${ }^{1}$ Hamid Reza Karimi, ${ }^{2}$ and Jianbin Qiu ${ }^{1}$ \\ ${ }^{1}$ Space Control and Inertial Technology Research Center, Harbin Institute of Technology, Harbin 150001, China \\ ${ }^{2}$ Faculty of Engineering and Science, University of Agder, N-4898 Grimstad, Norway \\ Correspondence should be addressed to Zhixiong Zhong; zhixiongzhong2012@gmail.com
}

Received 29 November 2012; Accepted 27 December 2012

Academic Editor: M. Chadli

Copyright (c) 2013 Zhixiong Zhong et al. This is an open access article distributed under the Creative Commons Attribution License, which permits unrestricted use, distribution, and reproduction in any medium, provided the original work is properly cited.

\begin{abstract}
The stability analysis and stabilization of Takagi-Sugeno (T-S) fuzzy delta operator systems with time-varying delay are investigated via an input-output approach. A model transformation method is employed to approximate the time-varying delay. The original system is transformed into a feedback interconnection form which has a forward subsystem with constant delays and a feedback one with uncertainties. By applying the scaled small gain (SSG) theorem to deal with this new system, and based on a Lyapunov Krasovskii functional (LKF) in delta operator domain, less conservative stability analysis and stabilization conditions are obtained. Numerical examples are provided to illustrate the advantages of the proposed method.
\end{abstract}

\section{Introduction}

The T-S fuzzy modeling approach, as a simple and effective tool for nonlinear control systems, has been widely accepted and extensive studied for a few decades [1-8]. In addition, it is well known that time delay is a source of instability or performance degradation [9]. Hence, analysis and synthesis of timedelay systems and other relative studies have attracted much attention during the past years [10-17]. Moreover, high-speed digital processing methods are of increasing importance in modern industrial applications. However, most traditional signal processing and control algorithms are inherently illconditioned when data are taken at high sampling rates [18]. The delta operator model can be applied as a useful approach to deal with discrete-time systems under high sampling rates through the analysis methods of continuous-time systems [19-22]. In view of the above considerations, both T-S fuzzy modeling approach and delta operator modeling approach have been extended to tackle the analysis and synthesis of nonlinear systems with time delay [23-25].

Recently, some works on analysis and design of T-S fuzzy systems via delta operator approach were developed [26-28]. However, to the authors' best knowledge, few results on the stability analysis and stabilization for Takagi-Sugeno (T-S) fuzzy delta operator systems with time-varying delay are proposed.

In this paper, an indirect approach, namely, the inputoutput (IO) approach is introduced to deal with the stability analysis and control design of T-S fuzzy delta operator systems with time-varying delay. The main contribution of paper is that the stability analysis and stabilization problems for fuzzy delta operator systems with time-varying delay are investigated by the IO approach. A model approximation method is employed to transform the original system into an equivalent interconnected system, which is comprised of a forward subsystem with constant time delays and a feedback one with delayed uncertainties. The scaled small gain (SSG) method is applied and an LKF in delta domain is constructed to analyze and synthesize this system. Furthermore, a frequency sweeping method [9] is suggested to guarantee the internal stability for the forward subsystem, such that less conservative results are ensured. Finally, some comparisons are made with the existing results and control of a trucktrailer model is also presented to illustrate the effectiveness of our method. 
This paper is organized as follows. A model transformation method and the proof of the SSG theorem for T-S fuzzy delta operator systems with time-varying delay are presented in Section 2. In Section 3, the stability analysis and stabilization results are provided. The simulation studies are given in Section 4 to illustrate the effectiveness of the proposed method. Finally, conclusions are drawn in Section 5.

Notations. The notations used throughout this paper are standard. $\mathbb{R}^{n}$ and $\mathbb{R}^{n \times m}$ represent the $n$-dimensional Euclidean space and $n \times m$ real matrices, respectively. $\mathbf{G}_{1} \circ \mathbf{G}_{2}$ represents the series connection of mapping $\mathbf{G}_{1}$ and $\mathbf{G}_{2}$. The notation $P>0(\geq 0)$ means that the matrix $P$ is positive (semi) definite, $I_{n}$ denotes an identity matrix with dimension $n$, and $\operatorname{diag}\{\cdots\}$ denotes a block-diagonal matrix. The symbol “*” in a matrix stands for the transposed elements in the symmetric positions.

\section{Model Description and Problem Formulation}

In the following, we consider a fuzzy delta operator system with time-varying delay, which can be described by the following T-S fuzzy model.

Plant Rule i. IF $\theta_{1}(t)$ is $M_{i 1}$ and $\theta_{2}(t)$ is $M_{i 2}$ and ... and $\theta_{p}(t)$ is $M_{i p}$, THEN

$$
\begin{aligned}
& \partial x(t)=A_{i} x(t)+A_{d i} x(t-n T)+B_{i} u(t), \\
& x(t)=\phi(t), \quad t \in\left[-h_{2}, 0\right], i=1,2, \ldots, r,
\end{aligned}
$$

where $x(t) \in \mathbb{R}^{n_{x}}$ is the state variable; $u(t) \in \mathbb{R}^{m}$ is control input; $n$ is a time-varying integer; $T$ is the sampling period; the bounded time-varying delay $n T$ satisfies $0<h_{1} \leq n T \leq$ $h_{2} ; \phi(t) \in \mathbb{R}^{n_{x}}$ is the vector-valued initial condition; $M_{i j}$ is the fuzzy set; $r$ is the number of IF-THEN rules; $\theta(t)=$ $\left[\theta_{1}(t), \theta_{2}(t), \ldots, \theta_{p}(t)\right]$ are the premise variables which do not depend on the control input; $A_{i}, A_{d i}$, and $B_{i}$ are known constant matrices with appropriate dimensions; $\partial x(t)$ is the delta operator of $x(t)$, which is defined by

$$
\partial x(t)= \begin{cases}\frac{d}{d t} x(t), & T=0, \\ \frac{x(t+T)-x(t)}{T}, & T \neq 0 .\end{cases}
$$

The overall T-S fuzzy delta operator system with timevarying delay is inferred as follows:

$$
\partial x(t)=\sum_{i=1}^{r} \lambda_{i}(\theta(t))\left[A_{i} x(t)+A_{d i} x(t-n T)+B_{i} u(t)\right]
$$

where $\sum_{i=1}^{r} \lambda_{i}(\theta(t))=1, \lambda_{i}(\theta(t))=\omega_{i}(\theta(t)) / \sum_{i=1}^{r} \omega_{i}(\theta(t)) \geq$ 0 , and $\omega_{i}(\theta(t))=\prod_{j=1}^{r} M_{i j}\left(\theta_{j}(t)\right)$ with $M_{i j}\left(\theta_{j}(t)\right)$ represent the grade of membership of $\theta_{j}(t)$ in $M_{i j}$.
The following control law is employed to deal with the problem of stabilization via state feedback, where the controller rule shares the same fuzzy sets with the T-S model.

Controller Rule $i$. IF $\theta_{1}(t)$ is $M_{i 1}$ and $\theta_{2}(t)$ is $M_{i 2}$ and ... and $\theta_{p}(t)$ is $M_{i p}$, THEN

$$
\begin{aligned}
u(t)= & K_{1 i} x(t)+\frac{1}{2} K_{2 i} x\left(t-h_{1}\right) \\
& +\frac{1}{2} K_{3 i} x\left(t-h_{2}\right), \quad i=1,2, \ldots, r .
\end{aligned}
$$

The overall T-S fuzzy state feedback control law is inferred as

$$
\begin{gathered}
u(t)=\sum_{i=1}^{r} \lambda_{i}(\theta(t))\left[K_{1 i} x(t)+\frac{1}{2} K_{2 i} x\left(t-h_{1}\right)\right. \\
\left.+\frac{1}{2} K_{3 i} x\left(t-h_{2}\right)\right] .
\end{gathered}
$$

Remark 1. It is noted that the controller given in (5) covers the special cases of the memoryless controller when $K_{2 i}=$ $K_{3 i}=0$ and the purely delayed controller when $K_{1 i}=0$, respectively.

Combining system (3) with the control law (5), the resulting closed-loop system can be expressed as follows:

$$
\begin{aligned}
\partial x(t)=\sum_{i=1}^{r} \sum_{j=1}^{r} & \lambda_{i}(\theta(t)) \lambda_{j}(\theta(t)) \\
\times & {\left[\left(A_{i}+B_{i} K_{1 j}\right) x(t)+A_{d i} x(t-n T)\right.} \\
& \left.\quad+\frac{1}{2} B_{i} K_{2 j} x\left(t-h_{1}\right)+\frac{1}{2} B_{i} K_{3 j} x\left(t-h_{2}\right)\right] .
\end{aligned}
$$

Before ending this section, we introduce the following lemmas as to be used to prove our main results in the following sections.

Lemma 2 (see [9]). Consider an interconnected system with two subsystems $\widetilde{\mathcal{S}}_{1}$ and $\widetilde{\mathcal{S}}_{2}$ :

$$
\begin{aligned}
& \widetilde{\mathcal{S}}_{1}: z(t)=\mathbf{G} \omega(t), \\
& \widetilde{\mathcal{S}}_{2}: \omega(t)=\Delta z(t),
\end{aligned}
$$

where the forward subsystem $\widetilde{\mathcal{S}}_{1}$ is known, the feedback subsystem $\mathcal{S}_{2}$ is unknown and time-varying, and assume that $\widetilde{\mathcal{S}}_{1}$ is internally stable. The closed-loop system formed by $\widetilde{\mathcal{S}}_{1}$ and $\widetilde{\mathcal{S}}_{2}$ is asymptotically stable for all $\Delta \in D \triangleq\left\{\Delta:\|\Delta\|_{\infty} \leq 1\right\}$ 
if there exist matrices $\left\{T_{w}, T_{z}\right\} \in \mathbb{T}$ satisfied:

$$
\begin{gathered}
\mathbb{T} \triangleq\left\{\left\{T_{w}, T_{z}\right\} \in \mathbb{R}^{w \times w} \times \mathbb{R}^{z \times z}: T_{w}, T_{z} \text { nonsigular } ;\right. \\
\left.\left\|T_{w} \circ \Delta \circ T_{z}^{-1}\right\|_{\infty} \leq 1\right\},
\end{gathered}
$$

such that the following SSG condition holds:

$$
\left\|T_{z} \circ \mathbf{G} \circ T_{w}^{-1}\right\|_{\infty} \leq 1 .
$$

Lemma 3 (see [29]). For any constant positive semidefinite symmetric matrix $W$, two positive integers $r$ and $r_{0}$ satifying $r \geq r_{0} \geq 1$, the following inequality holds:

$$
\left[\sum_{i=r_{0}}^{r} x(i)\right]^{T} W\left[\sum_{i=r_{0}}^{r} x(i)\right] \leq\left(r-r_{0}+1\right) \sum_{i=r_{0}}^{r} x^{T}(i) W x(i) .
$$

Lemma 4 (see [30]). The property of delta operator: for any time function $x(t)$ and $y(t)$, it holds that

$$
\partial(x(t) y(t))=\partial x(t) y(t)+x(t) \partial y(t)+T \partial x(t) \partial y(t),
$$

where $T$ is the sampling period.

\section{Model Transformation}

In this paper, the T-S fuzzy delta operator system with timevarying delay is investigated by an IO approach. By this method, the term $x(t-n T)$ is approximated and the error is written into the feedback path. The recent work in [31] proposed a two-term approximation method $(1 / 2)[x(t-$ $\left.\left.h_{1}\right)+x\left(t-h_{2}\right)\right]$ for $x(t-n T)$, which results in a smaller approximation error bound. Inspired by this method, the approximation error of time-varying delay can be expressed as

$$
\begin{aligned}
\omega_{t}(t) & =x(t-n T)-\frac{1}{2}\left[x\left(t-h_{1}\right)+x\left(t-h_{2}\right)\right] \\
& =\frac{T}{2} \sum_{i=-h_{2} / T}^{-n-1} \partial x(t+i T)-\frac{T}{2} \sum_{i=-n}^{-\left(h_{1} / T\right)-1} \partial x(t+i T) \\
& =\frac{T}{2} \sum_{i=-h_{2} / T}^{-\left(h_{1} / T\right)-1} k(i) \partial x(t+i T),
\end{aligned}
$$

where $\partial x(t)$ is defined in (2), and

$$
k(i)= \begin{cases}1, & i<-n \\ -1, & i \geq-n\end{cases}
$$

3.1. Open-Loop Case. Considering the fuzzy delta operator system (3) and setting $u(t)=0$, we have

$$
\partial x(t)=\sum_{i=1}^{r} \lambda_{i}(\theta(t))\left[A_{i} x(t)+A_{d i} x(t-n T)\right] .
$$

Employing the two-term approximation method to pull out the uncertainties of time-varying delay, the open-loop system can be written as an interconnected system with a forward subsystem and a feedback one, which is described by

$$
\begin{aligned}
& \mathcal{S}_{1}:\left[\frac{\partial x(t)}{z(t)}\right] \\
& =\sum_{i=1}^{r} \lambda_{i}(\theta(t))\left[\begin{array}{l|l}
\Theta_{1} & \frac{h_{12}}{2} A_{d i} X^{-1} \\
\hline X \Theta_{1} & X \frac{h_{12}}{2} A_{d i} X^{-1}
\end{array}\right] \\
& \quad \times\left[\frac{\zeta(t)}{\omega \omega(t)}\right], \\
& \mathcal{S}_{2}: \omega(t)=X \Delta X^{-1} z(t),
\end{aligned}
$$

where $\Theta_{1}=\left[\begin{array}{ll}A_{i} & (1 / 2) A_{d i} \\ (1 / 2) A_{d i}\end{array}\right], \zeta(t)=\operatorname{col}\{x(t) \quad x(t-$ $\left.\left.h_{1}\right) x\left(t-h_{2}\right)\right\}, h_{12}=h_{2}-h_{1}, \omega(t)=\left(2 / h_{12}\right) X \omega_{t}(t)$, the scaling matrix $\{X, X\} \in \mathbb{T}$ has the appropriate dimensions, and the operator $\Delta$ is the maping $z(t) \rightarrow \omega(t)$.

For convenience, we denote $\omega(t)=X \widetilde{\omega}(t)$ and $z(t)=$ $X \widetilde{z}(t)$. The system (15) can be rewritten as

$$
\begin{aligned}
& \mathcal{S}_{3}:\left[\begin{array}{c}
\partial x(t) \\
\widetilde{z}(t)
\end{array}\right] \\
& \quad=\sum_{i=1}^{r} \lambda_{i}(\theta(t))\left[\begin{array}{c|c}
\Theta_{1} & \frac{h_{12}}{2} A_{d i} \\
\hline \Theta_{1} & \frac{h_{12}}{2} A_{d i}
\end{array}\right]\left[\begin{array}{c}
\zeta(t) \\
\widetilde{\omega}(t)
\end{array}\right], \\
& \mathcal{S}_{4}: \widetilde{\omega}(t)=\Delta \widetilde{z}(t) .
\end{aligned}
$$

Now, the uncertainties of the time-varying delay have been pulled out from the system (14). Furthermore, the system has been transformed into the interconnection by the forward subsystem and the feedback subsystem. The following result shows that this reformulated system satisfies the following SSG condition.

Lemma 5. The operator $\Delta: z(t) \rightarrow \omega(t)$ in system (15) satisfies the SSG theorem if there exists the nosingular matrix $\{X, X\} \in \mathbb{T}$, such that

$$
\left\|X \Delta X^{-1}\right\| \leq 1
$$

Proof. Following the notations in (12), under the zero initial condition, we have the following inequalities by using the 
discrete Jensen inequality in Lemma 3:

$$
\begin{aligned}
\sum_{t=0}^{\infty} \omega^{T}(t) \omega(t) & \left(\frac{T}{h_{12}}\right)^{2} \sum_{t=0}^{\infty}\left[\sum_{i=-h_{2} / T}^{-\left(h_{1} / T\right)-1} k(i) \partial x(t+i T)\right]^{T} \\
& \times X^{T} X\left[\sum_{i=-h_{2} / T}^{-\left(h_{1} / T\right)-1} k(i) \partial x(t+i T)\right] \\
\leq & \frac{T}{h_{12}} \sum_{i=-h_{2} / T}^{-\left(h_{1} / T\right)-1} \sum_{t=0}^{\infty}\left[\partial x^{T}(t+i T) X^{T} X \partial x(t+i T)\right] \\
\leq & \frac{T}{h_{12}} \sum_{i=-h_{2} / T}^{-\left(h_{1} / T\right)-1} \sum_{t=0}^{\infty}\left[\partial x^{T}(t) X^{T} X \partial x(t)\right] \\
= & \sum_{t=0}^{\infty} z^{T}{ }_{(t)} z(t),
\end{aligned}
$$

which implies that $\left\|X \Delta X^{-1}\right\| \leq 1$. The proof is completed.

3.2. Closed-Loop Case. Employing the two-term approximation method to pull out the uncertainties of time-varying delay, the closed-loop system (6) can also be written as an interconnected system with a forward subsystem and a feedback one, which is described by

$$
\begin{aligned}
& \mathcal{S}_{5}:\left[\begin{array}{c}
\partial x(t) \\
\hline z(t)
\end{array}\right] \\
& =\sum_{i=1}^{r} \sum_{j=1}^{r} \lambda_{i}(\theta(t)) \lambda_{j}(\theta(t))\left[\begin{array}{l|l}
\Theta_{2} & \frac{h_{12}}{2} A_{d i} X^{-1} \\
\hline X \Theta_{2} & X \frac{h_{12}}{2} A_{d i} X^{-1}
\end{array}\right] \\
& \quad \times\left[\begin{array}{c}
\zeta(t) \\
\hline \widetilde{\omega}(t)
\end{array}\right], \\
& \mathcal{S}_{6}: \omega(t)=X \Delta X^{-1} z(t),
\end{aligned}
$$

where $\Theta_{2}=\left[\left(A_{i}+B_{i} K_{1 j}\right) \quad(1 / 2)\left(A_{d i}+B_{i} K_{2 j}\right) \quad(1 / 2)\left(A_{d i}+\right.\right.$ $\left.\left.B_{i} K_{3 j}\right)\right]$, and $\zeta(t), \omega(t)$, and $z(t)$ are defined as the same as the open-loop case.
For convenience, we denote $\omega(t)=X \widetilde{\omega}(t)$ and $z(t)=$ $X \widetilde{z}(t)$. The system (19) can be rewritten as

$$
\begin{aligned}
& \mathcal{S}_{7}:\left[\begin{array}{c}
\partial x(t) \\
\widetilde{z}(t)
\end{array}\right] \\
& \quad=\sum_{i=1}^{r} \sum_{j=1}^{r} \lambda_{i}(\theta(t)) \lambda_{j}(\theta(t))\left[\begin{array}{c|c}
\Theta_{2} & \frac{h_{12}}{2} A_{d i} \\
\hline \Theta_{2} & \frac{h_{12}}{2} A_{d i}
\end{array}\right]\left[\begin{array}{c}
\zeta(t) \\
\widetilde{\omega}(t)
\end{array}\right], \\
& \mathcal{S}_{8}: \widetilde{\omega}(t)=\Delta \widetilde{z}(t) .
\end{aligned}
$$

Remark 6. The definitions of $\omega(t)$ and $z(t)$ for the closedloop system are the same as the open-loop system, so it is easy to see that the closed-loop system (19) also satisfies the SSG condition.

Now the reformulated systems have been shown to satisfy the SSG condition in both the open-loop and closed-loop cases. Then the systems in (15) and (19) are asymptotically stable if both the forward subsystems are internally stable. Indeed, a frequency sweeping method is often used to check this condition [9].

Lemma 7 (see [9]). Consider the following system:

$$
\begin{aligned}
& \widetilde{\mathcal{S}}_{1}: z(t)=\mathbf{G} \omega(t), \\
& \widetilde{\mathcal{S}}_{2}: \omega(t)=\Delta z(t) .
\end{aligned}
$$

The aforementioned system is internally asymptotically stable if there exist a scalar $\varepsilon>0$ and a Lyapunov Krasovskii functional $V(t)$ satisfying

$$
V(t)>\varepsilon\|x(t)\|^{2},
$$

such that the functional

$$
w(t)=\dot{V}(t)+z^{T}(t) z(t)-\omega^{T}(t) \omega(t)
$$

statisfies

$$
w(t) \leq-\varepsilon\|x(t)\|^{2}-\varepsilon\|\omega(t)\|^{2} .
$$

\section{Stability Analysis}

The previous section presents a model transformation for the original system (3). The open-loop system has been converted into an interconnected system in (15), and the closed-loop system has been converted into (19). In this section, we investigate the asymptotic stability of the system in (15). First, we present the following result for T-S fuzzy delta system with time-varying delay.

Theorem 8. Consider T-S fuzzy delta operator system in (14). Then given scalars $h_{2}>h_{1}>0$ and the sampling period $T>0$, 
the fuzzy delta operator system (14) with time-varying delay is asymptotically stable if there exist positive definite symmetric matrices $U, P, R_{1}, R_{2}, Q_{1}, Q_{2}$, and $Z$, such that the following LMIs hold for $i=1,2, \ldots, r$ :

$$
\begin{aligned}
& \Phi_{i} \\
& =\left[\begin{array}{ccccc}
\Phi_{i}(1,1) & P A_{i} & \frac{1}{2} P A_{d i} & \frac{1}{2} P A_{d i} & \frac{h_{12}}{2} P A_{d i} \\
* & \Phi_{i}(2,2) & \frac{1}{2} P A_{d i}+\frac{R_{1}}{h_{1}} & \frac{1}{2} P A_{d i}+\frac{R_{2}}{h_{2}} & \frac{h_{12}}{2} P A_{d i} \\
* & * & -Q_{1}-\frac{1}{4} Z-\frac{R_{1}}{h_{1}} & -\frac{1}{4} Z & -\frac{h_{12}}{4} Z \\
* & * & * & -Q_{2}-\frac{1}{4} Z-\frac{R_{2}}{h_{2}} & -\frac{h_{12}}{4} Z \\
* & * & * & * & -\frac{h_{12}^{2}}{4} Z-U
\end{array}\right]
\end{aligned}
$$$$
<0 \text {, }
$$

where

$$
\begin{gathered}
\Phi_{i}(1,1)=T P+h_{1} R_{1}+h_{2} R_{2}-2 P+U \\
\Phi_{i}(2,2)=P A_{i}+A_{i}^{T} P+Z+\frac{h_{12}}{T} Z \\
+Q_{1}+Q_{2}-\frac{R_{1}}{h_{1}}-\frac{R_{2}}{h_{2}} .
\end{gathered}
$$

Proof. Firstly, choosing a Lyapunov-Krasovskii functional candidate in delta domain,

$$
V(t)=V_{1}(t)+V_{2}(t)+V_{3}(t)+V_{4}(t)
$$

where

$$
\begin{gathered}
V_{1}(t)=x^{T}(t) P x(t), \\
V_{2}(t)=T \sum_{i=h_{1} / T}^{h_{2} / T} \sum_{j=1}^{i} x^{T}(t-j T) Z x(t-j T), \\
V_{3}(t)=T \sum_{i=1}^{h_{1} / T} x^{T}(t-i T) Q_{1} x(t-i T) \\
+T \sum_{i=1}^{h_{2} / T} x^{T}(t-i T) Q_{2} x(t-i T), \\
V_{4}(t)=\sum_{i=1}^{h_{1} / T} \sum_{j=1}^{i} e^{T}(t-j T) R_{1} e(t-j T) \\
+\sum_{i=1}^{h_{2} / T} \sum_{j=1}^{i} e^{T}(t-j T) R_{2} e(t-j T),
\end{gathered}
$$

and $T$ is the sampling period, $e(j)=x(j)-x(j+T)$, so that $\partial x(j)=-e(j) / T$ and $e(t-j T)=x(t-j T)-x(t-(j-1) T)$.
Taking the delta operator manipulations of $V_{1}(t)$ along the trajectory of systems $\delta_{1}$ and $\delta_{2}$, and using Lemma 4 , it can be obtained that

$$
\begin{aligned}
& \partial V_{1}(t)=\partial^{T}(x(t)) P x(t)+x^{T}(t) P \partial(x(t)) \\
& +T \cdot \partial^{T}(x(t)) P \partial(x(t)) \\
& =\left[\begin{array}{c}
\partial x(t) \\
x(t) \\
x\left(t-h_{1}\right) \\
x\left(t-h_{2}\right) \\
\widetilde{\omega}(t)
\end{array}\right]^{T} \\
& \times\left[\begin{array}{ccccc}
T P & 0 & 0 & 0 & 0 \\
* & P A_{i}+A_{i}^{T} P & \frac{1}{2} P A_{d i} & \frac{1}{2} P A_{d i} & \frac{h_{12}}{2} P A_{d i} \\
* & * & 0 & 0 & 0 \\
* & * & * & 0 & 0 \\
* & * & * & * & 0
\end{array}\right] \\
& \times\left[\begin{array}{c}
\partial x(t) \\
x(t) \\
x\left(t-h_{1}\right) \\
x\left(t-h_{2}\right) \\
\widetilde{\omega}(t)
\end{array}\right] .
\end{aligned}
$$

Taking the delta operator manipulation of $V_{2}(t)$, we have

$$
\begin{aligned}
\partial V_{2}(t)= & T \cdot \frac{1}{T} \sum_{i=h_{1} / T}^{h_{2} / T} \sum_{j=1}^{i} x^{T}(t-(j-1) T) \\
& \times Z x(t-(j-1) T) \\
& -T \cdot \frac{1}{T} \sum_{i=h_{1} / T}^{h_{2} / T} \sum_{j=1}^{i} x^{T}(t-i T) Z x(t-i T) \\
= & \sum_{i=h_{1} / T}^{h_{2} / T} x^{T}(t) Z x(t) \\
& -\sum_{i=h_{1} / T}^{h_{2} / T} x^{T}(t-i T) Z x(t-i T) \\
\leq & \left(\frac{h_{12}}{T}+1\right) x^{T}(t) Z x(t) \\
& -x^{T}(t-n T) Z x(t-n T) .
\end{aligned}
$$


Substituting (12) into (30), we have

$$
\begin{aligned}
\partial V_{2}(t)= & {\left[\begin{array}{c}
x(t) \\
x\left(t-h_{1}\right) \\
x\left(t-h_{2}\right) \\
\widetilde{\omega}(t)
\end{array}\right]^{T} } \\
& \times\left[\begin{array}{cccc}
Z+\frac{h_{12}}{T} Z & 0 & 0 & 0 \\
* & -\frac{1}{4} Z & -\frac{1}{4} Z & -\frac{h_{12}}{4} Z \\
* & * & -\frac{1}{4} Z & -\frac{h_{12}}{4} Z \\
* & * & * & -\frac{h_{12}^{2}}{4} Z
\end{array}\right] \\
& \times\left[\begin{array}{c}
x(t) \\
x\left(t-h_{1}\right) \\
x\left(t-h_{2}\right) \\
\widetilde{\omega}(t)
\end{array}\right] .
\end{aligned}
$$

Taking the delta operator manipulation of $V_{3}(t)$, we have

$$
\begin{gathered}
\partial V_{3}(t)=\frac{1}{T} \cdot T\left[\sum_{i=1}^{h_{1} / T} x^{T}(t-(i-1) T)\right. \\
\times Q_{1} x(t-(i-1) T) \\
+\sum_{i=1}^{h_{2} / T} x^{T}(t-(i-1) T) \\
\times Q_{2} x(t-(i-1) T) \\
-\sum_{i=1}^{h_{1} / T} x^{T}(t-i T) Q_{1} x(t-i T) \\
\left.-\sum_{i=1}^{h_{2} / T} x^{T}(t-i T) Q_{2} x(t-i T)\right] \\
=x^{T}(t)\left(Q_{1}+Q_{2}\right) x(t) \\
-x^{T}\left(t-h_{1}\right) Q_{1} x\left(t-h_{1}\right) \\
-x^{T}\left(t-h_{2}\right) Q_{2} x\left(t-h_{2}\right) .
\end{gathered}
$$

Taking the delta operator manipulation of $V_{4}(t)$ and using Lemma 3, we have

$$
\begin{array}{r}
\partial V_{4}(t)=\frac{1}{T}\left[\sum_{i=1}^{h_{1} / T} \sum_{j=1}^{i} e^{T}(t-(j-1) T)\right. \\
\times R_{1} e(t-(j-1) T)
\end{array}
$$

$$
\begin{gathered}
+\sum_{i=1}^{h_{2} / T} \sum_{j=1}^{i} e^{T}(t-(j-1) T) \\
\times R_{2} e(t-(j-1) T) \\
-\sum_{i=1}^{h_{1} / T} \sum_{j=1}^{i} e^{T}(t-j T) R_{1} e(t-j T) \\
\left.-\sum_{i=1}^{h_{2} / T} \sum_{j=1}^{i} e^{T}(t-j T) R_{2} e(t-j T)\right]
\end{gathered}
$$$$
\leq \frac{h_{1}}{T^{2}} e^{T}(t) R_{1} e(t)
$$$$
-\frac{1}{h_{1}}\left(\sum_{i=1}^{h_{1} / T} e(t-i T)\right)^{T} R_{1}\left(\sum_{i=1}^{h_{1} / T} e(t-i T)\right)
$$$$
+\frac{h_{2}}{T^{2}} e^{T}(t) R_{2} e(t)
$$$$
-\frac{1}{h_{2}}\left(\sum_{i=1}^{h_{2} / T} e(t-i T)\right)^{T} R_{2}\left(\sum_{i=1}^{h_{2} / T} e(t-i T)\right)
$$$$
=h_{1} \partial x^{T}(t) R_{1} \partial x(t)-\frac{1}{h_{1}}\left(x\left(t-h_{1}\right)-x(t)\right)^{T}
$$$$
\times R_{1}\left(x\left(t-h_{1}\right)-x(t)\right)+h_{2} \partial x^{T}(t) R_{2} \partial x(t)
$$$$
-\frac{1}{h_{2}}\left(x\left(t-h_{2}\right)-x(t)\right)^{T} R_{2}\left(x\left(t-h_{2}\right)-x(t)\right) \text {. }
$$

For the positive definite symmetric matrix $P$, we have the following equation from (16):

$$
\begin{aligned}
0=-\sum_{i=1}^{r} \lambda_{i}(\theta(t)) 2 \partial^{T}(x(t)) P & \\
\times & {\left[\partial x(t)-A_{i} x(t)-\frac{1}{2} A_{d i}\left(x\left(t-h_{1}\right)+x\left(t-h_{2}\right)\right)\right.} \\
& \left.-\frac{h_{12}}{2} A_{d i} \widetilde{\omega}(t)\right] .
\end{aligned}
$$

Substituting (34) into $\partial V(t)$, we have

$$
\partial V(t)=\sum_{i=1}^{r} \lambda_{i}(\theta(t)) \xi^{T} \Sigma_{1 i} \xi
$$


where

$$
\begin{aligned}
& \Sigma_{1 i} \\
& =\left[\begin{array}{ccccc}
\Sigma_{1 i}(1,1) & P A_{i} & \frac{1}{2} P A_{d i} & \frac{1}{2} P A_{d i} & \frac{h_{12}}{2} P A_{d i} \\
* & \Sigma_{1 i}(2,2) & \frac{1}{2} P A_{d i}+\frac{R_{1}}{h_{1}} & \frac{1}{2} P A_{d i}+\frac{R_{2}}{h_{2}} & \frac{h_{12}}{2} P A_{d i} \\
* & * & -Q_{1}-\frac{1}{4} Z-\frac{R_{1}}{h_{1}} & -\frac{1}{4} Z & -\frac{h_{12}}{4} Z \\
* & * & * & -Q_{2}-\frac{1}{4} Z-\frac{R_{2}}{h_{2}} & -\frac{h_{12}}{4} Z \\
* & * & * & * & -\frac{h_{12}^{2}}{4} Z
\end{array}\right], \\
& \Sigma_{1 i}(1,1)=T P+h_{1} R_{1}+h_{2} R_{2}-2 P, \\
& \Sigma_{1 i}(2,2)=P A_{i}+A_{i}^{T} P+Z+\frac{h_{12}}{T} Z+Q_{1}+Q_{2}-\frac{R_{1}}{h_{1}}-\frac{R_{2}}{h_{2}}, \\
& \xi^{T}=\left[\partial^{T}(x(t)) x^{T}(t) x^{T}\left(t-h_{1}\right) x^{T}\left(t-h_{2}\right) \widetilde{\omega}^{T}(t)\right] .
\end{aligned}
$$

Therefore if $\partial V(t)<0$, there always exists a sufficiently small scalar $\varepsilon$, for $x(t) \neq 0$, such that $\partial V(t) \leq-\varepsilon\|x(t)\|^{2}$, which indicates that the systems $\mathcal{S}_{1}$ and $\mathcal{S}_{2}$ under $\omega(t)=0$ are asymptotically stable.

Next, to consider the condition $\omega(t) \neq 0$, we denote $U=$ $X^{T} X>0$ and it can be expanded in Lemma 7 as

$$
\begin{aligned}
\mathscr{W} \triangleq & \partial V(t)+z^{T}(t) z(t)-\omega^{T}(t) \omega(t) \\
= & \sum_{i=1}^{r} \lambda_{i}(\theta(t)) \xi^{T} \Sigma_{1 i} \xi+\widetilde{z}^{T}(t) X^{T} X \widetilde{z}(t) \\
& -\widetilde{\omega}^{T}(t) X^{T} X \widetilde{\omega}(t) \\
= & \sum_{i=1}^{r} \lambda_{i}(\theta(t)) \xi^{T} \Sigma_{2 i} \xi,
\end{aligned}
$$

where $\Sigma_{2 i}=\Phi_{i}$. The proof is completed.

To compare the results obtained by IO approach, we give the following corollary, which is obtained by a direct LKFbased method.

Corollary 9. Consider T-S fuzzy delta operator system in (14). Then given scalars $h_{2}>h_{1}>0$ and the sampling period $T>0$, the fuzzy delta operator system (14) with time-varying delay is asymptotically stable if there exist positive definite symmetric matrices $P, R_{1}, R_{2}, Q_{1}, Q_{2}$, and $Z$, matrices $N=\left[\begin{array}{l}N_{1} \\ N_{2}\end{array}\right], M=$ $\left[\begin{array}{l}M_{1} \\ M_{2}\end{array}\right], S=\left[\begin{array}{l}S_{1} \\ S_{2}\end{array}\right], \widetilde{X}=\left[\begin{array}{ll}\widetilde{X}_{11} & \widetilde{X}_{12} \\ \widetilde{X}_{12}^{T} & \widetilde{X}_{22}\end{array}\right]$, and $Y=\left[\begin{array}{ll}Y_{11} & Y_{12} \\ Y_{12}^{T} & Y_{22}\end{array}\right]$, such that the following LMIs (38)-(39) hold:

$$
\Psi_{1}=\left[\begin{array}{ccc}
-\widetilde{X}_{11} & -\widetilde{X}_{12} & N_{1} \\
* & -\widetilde{X}_{22} & N_{2} \\
* & * & -\frac{R_{2}}{T}
\end{array}\right]<0,
$$

$$
\begin{gathered}
\Psi_{2}=\left[\begin{array}{ccc}
-\widetilde{X}_{11} & -\widetilde{X}_{12} & M_{1} \\
* & -\widetilde{X}_{22} & M_{2} \\
* & * & -\frac{R_{2}}{T}
\end{array}\right]<0, \\
\Psi_{3}=\left[\begin{array}{ccc}
-Y_{11} & -Y_{12} & S_{1} \\
* & -Y_{22} & S_{2} \\
* & * & -\frac{R_{1}}{T}
\end{array}\right]<0, \\
\Psi_{4 i}=\left[\begin{array}{ccccc}
\Psi_{4 i}(1,1) & P A_{i} & P A_{d i} & 0 & 0 \\
* & \Psi_{4 i}(2,2) & \Psi_{4 i}(2,3) & -S_{1} & -M_{1} \\
* & * & \Psi_{4 i}(3,3) & -S_{2} & -M_{2} \\
* & * & * & -Q_{1} & 0 \\
* & * & * & * & -Q_{2}
\end{array}\right] \\
<0, \quad \begin{array}{llll}
* \\
i=1,2, \ldots,
\end{array}
\end{gathered}
$$

where

$$
\begin{gathered}
\Psi_{4 i}(1,1)=T P+h_{1} R_{1}+h_{2} R_{2}-2 P, \\
\Psi_{4 i}(2,2)=P A_{i}+A_{i}^{T} P+Z+\frac{h_{12}}{T} Z+Q_{1}+Q_{2} \\
+N_{1}+N_{1}^{T}+S_{1}+S_{1}^{T}+\frac{h_{2}}{T} \widetilde{X}_{11}+\frac{h_{1}}{T} Y_{11}, \\
\Psi_{4 i}(2,3)= \\
P A_{d i}-N_{1}+N_{2}^{T}+M_{1}+S_{2}^{T} \\
+\frac{h_{2}}{T} \widetilde{X}_{12}+\frac{h_{1}}{T} Y_{12}, \\
\Psi_{4 i}(3,3)=-Z-N_{2}-N_{2}^{T}+M_{2}+M_{2}^{T} \\
+\frac{h_{2}}{T} \widetilde{X}_{22}+\frac{h_{1}}{T} Y_{22} .
\end{gathered}
$$

Proof. To make a fair comparison, we choose the same LKF candidate as in the proof of Theorem 8 .

Taking the delta operator manipulations of $V_{1}(t), V_{2}(t)$, $V_{3}(t)$, and $V_{4}(t)$ along the trajectory of system (14), we have

$$
\begin{aligned}
\partial V_{1}(t)= & \partial^{T}(x(t)) P x(t)+x^{T}(t) P \partial(x(t)) \\
& +T \cdot \partial^{T}(x(t)) P \partial(x(t)) \\
= & {\left[\begin{array}{c}
\partial x(t) \\
x(t) \\
x(t-n T)
\end{array}\right]^{T}\left[\begin{array}{ccc}
T P & 0 & 0 \\
* & P A_{i}+A_{i}^{T} P & P A_{d i} \\
* & * & 0
\end{array}\right] } \\
& \times\left[\begin{array}{c}
\partial x(t) \\
x(t) \\
x(t-n T)
\end{array}\right],
\end{aligned}
$$




$$
\begin{aligned}
\partial V_{2}(t) \leq & \left(\frac{h_{12}}{T}+1\right) x^{T}(t) Z x(t) \\
& -x^{T}(t-n T) Z x(t-n T), \\
\partial V_{3}(t)= & x^{T}(t)\left(Q_{1}+Q_{2}\right) x(t) \\
& -x^{T}\left(t-h_{1}\right) Q_{1} x\left(t-h_{1}\right) \\
& -x^{T}\left(t-h_{2}\right) Q_{2} x\left(t-h_{2}\right), \\
\partial V_{4}(t)= & h_{1} \partial^{T}(x(t)) R_{1} \partial(x(t)) \\
& -\frac{1}{T} \sum_{i=1}^{h_{1} / T} e^{T}(t-i T) R_{1} e(t-i T) \\
& +h_{2} \partial^{T}(x(t)) R_{2} \partial(x(t)) \\
& -\frac{1}{T} \sum_{i=1}^{h_{2} / T} e^{T}(t-i T) R_{2} e(t-i T),
\end{aligned}
$$

where $e(t-i T)$ is defined in (27).

For a positive definite symmetric matrix $P$, we have the following equation from (14):

$$
\begin{aligned}
0=-\sum_{i=1}^{r} & \lambda_{i}(\theta(t)) 2 \partial^{T}(x(t)) P \\
& \times\left[\partial x(t)-A_{i} x(t)-A_{d i} x(t-n T)\right] .
\end{aligned}
$$

From the definition of $e(t-i T)$, the following equations hold for any matrices $N, M$, and $S$ with appropriate dimensions:

$$
\begin{gathered}
\begin{array}{r}
0 \Upsilon^{T}(t) N\left[x(t)-x(t-n T)+\sum_{i=1}^{n} e^{T}(t-i T)\right], \\
0=2 \Upsilon^{T}(t) M\left[x(t-n T)-x\left(t-h_{2}\right)\right. \\
\left.+\sum_{i=n+1}^{h_{2} / T} e^{T}(t-i T)\right], \\
0=2 \Upsilon^{T}(t) S\left[x(t)-x\left(t-h_{1}\right)+\sum_{i=1}^{h_{1} / T} e^{T}(t-i T)\right],
\end{array}
\end{gathered}
$$

where $\Upsilon^{T}(t)=\left[x^{T}(t) x^{T}(t-n T)\right]$.
For any appropriate dimensions matrices $\widetilde{X}=\widetilde{X}^{T}$ and $Y=Y^{T}$, we have

$$
\begin{aligned}
0= & \sum_{i=1}^{h_{2} / T} \Upsilon^{T}(t) \widetilde{X} \Upsilon(t)-\sum_{i=1}^{h_{2} / T} \Upsilon^{T}(t) \widetilde{X} \Upsilon(t) \\
= & \frac{h_{2}}{T} \Upsilon^{T}(t) \widetilde{X} \Upsilon(t)-\sum_{i=1}^{n} \Upsilon^{T}(t) \widetilde{X} \Upsilon(t) \\
& -\sum_{i=n+1}^{h_{2} / T} \Upsilon^{T}(t) \widetilde{X} \Upsilon(t), \\
0= & \sum_{i=1}^{h_{1} / T} \Upsilon^{T}(t) Y \Upsilon(t)-\sum_{i=1}^{h_{1} / T} \Upsilon^{T}(t) Y \Upsilon(t) \\
= & \frac{h_{1}}{T} \Upsilon^{T}(t) Y \Upsilon(t)-\sum_{i=1}^{h_{1} / T} \Upsilon^{T}(t) Y \Upsilon(t) .
\end{aligned}
$$

Substituting (42)-(44) into $\partial V(t)$, we have

$$
\begin{aligned}
\partial V(t)= & \sum_{i=1}^{r} \lambda_{i}(t) \xi_{1}^{T} \Sigma_{3 i} \xi_{1}+\sum_{i=1}^{n} \Upsilon_{1}^{T}(t) \Sigma_{4} \Upsilon_{1}(t) \\
& +\sum_{i=1}^{h_{1} / T} \Upsilon_{1}^{T}(t) \Sigma_{5} \Upsilon_{1}(t)+\sum_{i=n+1}^{h_{2} / T} \Upsilon_{1}^{T}(t) \Sigma_{6} \Upsilon_{1}(t)
\end{aligned}
$$

where $\xi_{1}^{T}=\left[\begin{array}{lllll}\partial x^{T}(t) & x^{T}(t) & x^{T}(t-n T) & x^{T}\left(t-h_{1}\right) & x^{T}\left(t-h_{2}\right)\end{array}\right]$, $\Upsilon_{1}^{T}(t)=\left[\begin{array}{lll}x^{T}(t) & x^{T}(t-n T) e^{T}(t-i T)\end{array}\right], \Sigma_{3 i}=\Psi_{4 i}, \Sigma_{4}=\Psi_{1}$, $\Sigma_{5}=\Psi_{3}$, and $\Sigma_{6}=\Psi_{2}$. Since $\Sigma_{3 i}<0, \Sigma_{4}<0, \Sigma_{5}<0$, and $\Sigma_{6}<0$ hold, then $\partial V(t)<0$. The proof is completed.

\section{Stabilization}

The previous section presents the criterion for asymptotic stability of fuzzy delta operator open-loop system. In this section, we are interested in designing a controller in (5). By employing the same LKF and applying IO method, the following criteria can be obtained.

Theorem 10. Consider T-S fuzzy delta operator system (3) with the controller in (5). Then given scalars $h_{2}>h_{1}>0$ and the sampling period $T>0$, the fuzzy delta operator system with time-varying delay is asymptotically stable if there exist positive definite symmetric matrices $G, \bar{U}, \bar{R}_{1}, \bar{R}_{2}, \bar{Q}_{1}, \bar{Q}_{2}$, and $\bar{Z}$ and matrices $\widetilde{K}_{1 i}, \widetilde{K}_{2 i}$, and $\widetilde{K}_{3 i}$, such that the following LMIs hold:

$$
\begin{array}{cl}
\Phi_{i i}<0, & (1 \leq i \leq r), \\
\Phi_{i j}+\Phi_{j i}<0, & (1 \leq i<j \leq r),
\end{array}
$$


where

$\Phi_{i j}$

$$
\begin{aligned}
& =\left[\begin{array}{ccccc}
\Phi_{i j}(1,1) & \Phi_{i j}(1,2) & \Phi_{i j}(1,3) & \Phi_{i j}(1,4) & \frac{h_{12}}{2} A_{d i} G \\
* & \Phi_{i j}(2,2) & \Phi_{i j}(2,3) & \Phi_{i j}(2,4) & \frac{h_{12}}{2} A_{d i} G \\
* & * & \Phi_{i j}(3,3) & -\frac{1}{4} \bar{Z} & -\frac{h_{12}}{4} \bar{Z} \\
* & * & * & \Phi_{i j}(4,4) & -\frac{h_{12}}{4} \bar{Z} \\
* & * & * & * & -\frac{h_{12}^{2}}{4} \bar{Z}-\bar{U}
\end{array}\right] \\
& \Phi_{i j}(1,1)=T G+h_{1} \bar{R}_{1}+h_{2} \bar{R}_{2}-2 G+\bar{U}, \\
& \Phi_{i j}(1,2)=A_{i} G+B_{i} \widetilde{K}_{1 j}, \\
& \Phi_{i j}(1,3)=\frac{1}{2}\left(A_{d i} G+B_{i} \widetilde{K}_{2 j}\right), \\
& \Phi_{i j}(1,4)=\frac{1}{2}\left(A_{d i} G+B_{i} \widetilde{K}_{3 j}\right), \\
& \Phi_{i j}(2,2)=A_{i} G+B_{i} \widetilde{K}_{1 j}+G A_{i}^{T}+\widetilde{K}_{1 j}^{T} B_{i}^{T} \\
& +\bar{Z}+\frac{h_{12}}{T} \bar{Z}+\bar{Q}_{1}+\bar{Q}_{2}-\frac{\bar{R}_{1}}{h_{1}}-\frac{\bar{R}_{2}}{h_{2}}, \\
& \Phi_{i j}(2,3)=\frac{1}{2}\left(A_{d i} G+B_{i} \widetilde{K}_{2 j}\right)+\frac{\bar{R}_{1}}{h_{1}}, \\
& \Phi_{i j}(2,4)=\frac{1}{2}\left(A_{d i} G+B_{i} \widetilde{K}_{3 j}\right)+\frac{\bar{R}_{2}}{h_{2}}, \\
& \Phi_{i j}(3,3)=-\bar{Q}_{1}-\frac{1}{4} \bar{Z}-\frac{\bar{R}_{1}}{h_{1}}, \\
& \Phi_{i j}(4,4)=-\bar{Q}_{2}-\frac{1}{4} \bar{Z}-\frac{\bar{R}_{2}}{h_{2}} .
\end{aligned}
$$

Moreover, a suitable stabilizing fuzzy state feedback controller can be chosen by

$$
\begin{aligned}
u(t)=\sum_{i=1}^{r} \lambda_{i}(\theta(t))[ & K_{1 i} x(t)+\frac{1}{2} K_{2 i} x\left(t-h_{1}\right) \\
& \left.+\frac{1}{2} K_{3 i} x\left(t-h_{2}\right)\right], \quad i=1,2, \ldots, r,
\end{aligned}
$$

where $K_{1 i}=\widetilde{K}_{1 i} G^{-1}, K_{2 i}=\widetilde{K}_{2 i} G^{-1}, K_{3 i}=\widetilde{K}_{3 i} G^{-1}$.

Proof. Choosing the same LKF candidate as in the proof of Theorem 8 , we have

$$
\partial V(t)=\sum_{i=1}^{r} \sum_{j=1}^{r} \lambda_{i}(\theta(t)) \lambda_{j}(\theta(t)) \xi^{T} \Sigma_{1 i j} \xi
$$

where

$$
\begin{aligned}
& \Sigma_{1 i j} \\
& =\left[\begin{array}{ccccc}
\Sigma_{1 i j}(1,1) & \Sigma_{1 i j}(1,2) & \Sigma_{1 i j}(1,3) & \Sigma_{1 i j}(1,4) & \frac{h_{12}}{2} P A_{d i} \\
* & \Sigma_{1 i j}(2,2) & \Sigma_{1 i j}(2,3) & \Sigma_{1 i j}(2,4) & \frac{h_{12}}{2} P A_{d i} \\
* & * & \Sigma_{1 i j}(3,3) & -\frac{1}{4} Z & -\frac{h_{12}}{4} Z \\
* & * & * & \Sigma_{1 i j}(4,4) & -\frac{h_{12}}{4} Z \\
* & * & * & * & -\frac{h_{12}^{2}}{4} Z
\end{array}\right], \\
& \Sigma_{1 i j}(1,1)=T P+h_{1} R_{1}+h_{2} R_{2}-2 P, \\
& \Sigma_{1 i j}(1,2)=P\left(A_{i}+B_{i} K_{1 j}\right), \\
& \Sigma_{1 i j}(1,3)=\frac{1}{2} P\left(A_{d i}+B_{i} K_{2 j}\right), \\
& \Sigma_{1 i j}(1,4)=\frac{1}{2} P\left(A_{d i}+B_{i} K_{3 j}\right), \\
& \Sigma_{1 i j}(2,2)=P\left(A_{i}+B_{i} K_{1 j}\right)+\left(A_{i}+B_{i} K_{1 j}\right)^{T} P \\
& +Z+\frac{h_{12}}{T} Z+Q_{1}+Q_{2}-\frac{R_{1}}{h_{1}}-\frac{R_{2}}{h_{2}}, \\
& \Sigma_{1 i j}(2,3)=\frac{1}{2} P\left(A_{d i}+B_{i} K_{2 j}\right)+\frac{R_{1}}{h_{1}}, \\
& \Sigma_{1 i j}(2,4)=\frac{1}{2} P\left(A_{d i}+B_{i} K_{3 j}\right)+\frac{R_{2}}{h_{2}}, \\
& \Sigma_{1 i j}(3,3)=-Q_{1}-\frac{1}{4} Z-\frac{R_{1}}{h_{1}}, \\
& \Sigma_{1 i j}(4,4)=-Q_{2}-\frac{1}{4} Z-\frac{R_{2}}{h_{2}},
\end{aligned}
$$

and $\xi$ is defined in (35)

Next, by applying Lemma 7, we have

$$
\begin{aligned}
\mathscr{W} \triangleq & \partial V(t)+z^{T}(t) z(t)-\omega^{T}(t) \omega(t) \\
= & \sum_{i=1}^{r} \sum_{j=1}^{r} \lambda_{i}(\theta(t)) \lambda_{j}(\theta(t)) \xi^{T} \Sigma_{1 i j} \xi \\
& +\widetilde{z}^{T}(t) X^{T} X \widetilde{z}(t)-\widetilde{\omega}^{T}(t) X^{T} X \widetilde{\omega}(t) \\
= & \sum_{i=1}^{r} \sum_{j=1}^{r} \lambda_{i}(\theta(t)) \lambda_{j}(\theta(t)) \xi^{T} \Sigma_{2 i j} \xi,
\end{aligned}
$$


where

$$
\begin{aligned}
& \Sigma_{2 i j} \\
& =\left[\begin{array}{ccccc}
\Sigma_{1 i j}(1,1)+U & \Sigma_{1 i j}(1,2) & \Sigma_{1 i j}(1,3) & \Sigma_{1 i j}(1,4) & \frac{h_{12}}{2} P A_{d i} \\
* & \Sigma_{1 i j}(2,2) & \Sigma_{1 i j}(2,3) & \Sigma_{1 i j}(2,4) & \frac{h_{12}}{2} P A_{d i} \\
* & * & \Sigma_{1 i j}(3,3) & -\frac{1}{4} Z & -\frac{h_{12}}{4} Z \\
* & * & * & \Sigma_{1 i j}(4,4) & -\frac{h_{12}}{4} Z \\
* & * & * & * & -\frac{h_{12}^{2}}{4} Z-U
\end{array}\right] .
\end{aligned}
$$

It can be clearly shown that

$$
\begin{aligned}
\mathscr{W} \triangleq & \sum_{i=1}^{r} \lambda_{i}^{2}(\theta(t)) \xi^{T} \Sigma_{2 i i} \xi \\
& +\sum_{i=1}^{r} \sum_{i<j}^{r} \lambda_{i}(\theta(t)) \lambda_{j}(\theta(t)) \xi^{T} \times\left(\Sigma_{2 i j}+\Sigma_{2 j i}\right) \xi .
\end{aligned}
$$

Premultiplying and postmultiplying $\Sigma_{2 i i}$ by $\operatorname{diag}\left\{P^{-1} P^{-1} P^{-1} P^{-1} P^{-1}\right\}$, and letting $G=P^{-1}, \bar{R}_{1}=$ $P^{-1} R_{1} P^{-1}, \bar{R}_{2}=P^{-1} R_{2} P^{-1}, \bar{Q}_{1}=P^{-1} Q_{1} P^{-1}, \bar{Q}_{2}=P^{-1} Q_{2} P^{-1}$, $\bar{U}=P^{-1} U P^{-1}, \widetilde{K}_{1 i}=K_{1 i} P^{-1}, \widetilde{K}_{2 i}=K_{2 i} P^{-1}$, and $\widetilde{K}_{3 i}=K_{3 i} P^{-1}$ yield $\Phi_{i i}$. Following a similar line in the previous process to $\Sigma_{2 i j}$ and $\Sigma_{2 j i}$ yields $\Phi_{i j}$ and $\Phi_{j i}$.

Since $\Phi_{i i}<0$ holds for $1 \leq i \leq r$, and $\left(\Phi_{i j}+\Phi_{j i}\right)<0$ holds for $1 \leq i<j \leq r$, then we have $\mathscr{W}<0$. Then by using Lemma 7, the system (19) is internally asymptotically stable. Furthermore, from Lemma 2, the fuzzy delta operator system (3) under the controller (5) is asymptotically stable. Finally, the explicit expression of the state feedback controller is given by $K_{1 i}=\widetilde{K}_{1 i} G^{-1}, K_{2 i}=\widetilde{K}_{2 i} G^{-1}$, and $K_{3 i}=\widetilde{K}_{3 i} G^{-1}$. The proof is completed.

To compare the results obtained by the IO approach, we give the following corollary, which is obtained by a direct LKF-based method.

Corollary 11. Consider T-S fuzzy delta operator system (3) with the controller in (5). Then given scalars $h_{2}>h_{1}>0$ and the sampling period $T>0$, the fuzzy delta operator system with time-varying delay is asymptotically stable if there exist positive definite symmetric matrices $G, \bar{R}_{1}, \bar{R}_{2}, \bar{Q}_{1}, \bar{Q}_{2}$, and $\bar{Z}$ and matrices $\bar{N}=\left[\begin{array}{l}\bar{N}_{1} \\ \bar{N}_{2}\end{array}\right], \bar{M}=\left[\begin{array}{l}\bar{M}_{1} \\ \bar{M}_{2}\end{array}\right], \bar{S}=\left[\begin{array}{l}\bar{S}_{1} \\ \bar{S}_{2}\end{array}\right], \bar{X}=\left[\begin{array}{ll}\bar{X}_{11} & \bar{X}_{12} \\ \bar{X}_{12}^{T} & \bar{X}_{22}\end{array}\right]$, $\bar{Y}=\left[\begin{array}{ll}\bar{Y}_{11} & \bar{Y}_{12} \\ \bar{Y}_{12}^{T} & \bar{Y}_{22}\end{array}\right]$ and $\widetilde{K}_{1 i}$, such that (38) and the following LMIs hold:

$$
\begin{array}{cl}
\Psi_{4 i i}<0 & (1 \leq i \leq r), \\
\Psi_{4 i j}+\Psi_{4 j i}<0 & (1 \leq i<j \leq r),
\end{array}
$$

where

$$
\begin{gathered}
\Psi_{4 i j}=\left[\begin{array}{ccccc}
\Psi_{4 i j}(1,1) & A_{i} G+B_{i} \widetilde{K}_{1 j} & A_{d i} G & 0 & 0 \\
* & \Psi_{4 i j}(2,2) & \Psi_{4 i j}(2,3) & -\bar{S}_{1} & -\bar{M}_{1} \\
* & * & \Psi_{4 i j}(3,3) & -\bar{S}_{2} & -\bar{M}_{2} \\
* & * & * & -\bar{Q}_{1} & 0 \\
* & * & * & * & -\bar{Q}_{2}
\end{array}\right] \\
\Psi_{4 i j}(2,2)=A_{i j}(1,1)=T G+h_{1} \bar{R}_{1}+h_{2} \bar{R}_{2}-2 G \\
+\frac{h_{12}}{T} \bar{Z}+\bar{K}_{1 j}+G A_{i}^{T}+\bar{K}_{1 j}^{T} B_{i}^{T}+\bar{Z}_{2}+\bar{N}_{1}+\bar{N}_{1}^{T}+\bar{S}_{1}+\bar{S}_{1}^{T} \\
+\frac{h_{2}}{T} \bar{X}_{11}+\frac{h_{1}}{T} \bar{Y}_{11}, \\
\Psi_{4 i j}(2,3)=A_{d i} G-\bar{N}_{1}+\bar{N}_{2}^{T}+\bar{M}_{1}+\bar{S}_{2}^{T} \\
+\frac{h_{2}}{T} \bar{X}_{12}+\frac{h_{1}}{T} \bar{Y}_{12}, \\
\Psi_{4 i j}(3,3)=-\frac{h_{2}}{T} \bar{X}_{22}+\frac{h_{1}}{T} \bar{Y}_{22} . \\
\bar{N}_{2}-\bar{N}_{2}^{T}+\bar{M}_{2}+\bar{M}_{2}^{T}
\end{gathered}
$$

Moreover, a suitable stabilizing fuzzy state feedback controller is given by

$$
u(t)=\sum_{i=1}^{r} \lambda_{i}(\theta(t)) K_{1 i} x(t), \quad i=1,2, \ldots, r
$$

where $K_{1 i}=\widetilde{K}_{1 i} G^{-1}$.

Proof. Choosing the same LKF candidate as in the proof of Theorem 8, we have

$$
\begin{aligned}
\partial V(t)= & \sum_{i=1}^{r} \sum_{j=1}^{r} \lambda_{i}(\theta(t)) \lambda_{j}(\theta(t)) \xi_{1}^{T} \Sigma_{3 i j} \xi_{1} \\
& +\sum_{i=1}^{n} \Upsilon_{1}^{T}(t) \Sigma_{4} \Upsilon_{1}(t)+\sum_{i=1}^{h_{1} / T} \Upsilon_{1}^{T}(t) \Sigma_{5} \Upsilon_{1}(t) \\
& +\sum_{i=n+1}^{h_{2} / T} \Upsilon_{1}^{T}(t) \Sigma_{6} \Upsilon_{1}(t),
\end{aligned}
$$


where

$$
\begin{gathered}
\Sigma_{3 i j}=\left[\begin{array}{ccccc}
\Sigma_{3 i j}(1,1) & P\left(A_{i}+B_{i} K_{1 j}\right) & P A_{d i} & 0 & 0 \\
* & \Sigma_{3 i j}(2,2) & \Sigma_{3 i j}(2,3) & -S_{1} & -M_{1} \\
* & * & \Sigma_{3 i j}(3,3) & -S_{2} & -M_{2} \\
* & * & * & -Q_{1} & 0 \\
* & * & * & * & -Q_{2}
\end{array}\right], \\
\Sigma_{3 i j}(1,1)=T P+h_{1} R_{1}+h_{2} R_{2}-2 P \\
\Sigma_{3 i j}(2,2)=P\left(A_{i}+B_{i} K_{1 j}\right)+\left(A_{i}+B_{i} K_{1 j}\right)^{T} P+Z \\
+\frac{h_{12}}{T} Z+Q_{1}+Q_{2}+N_{1}+N_{1}^{T}+S_{1}+S_{1}^{T} \\
+\frac{h_{2}}{T} \widetilde{X}_{11}+\frac{h_{1}}{T} Y_{11}, \\
\Sigma_{3 i j}(2,3)=P A_{d i}-N_{1}+N_{2}^{T}+M_{1}+S_{2}^{T} \\
+\frac{h_{2}}{T} \widetilde{X}_{12}+\frac{h_{1}}{T} Y_{12}, \\
\quad+\frac{h_{2}}{T} \widetilde{X}_{22}+\frac{h_{1}}{T} Y_{22},
\end{gathered}
$$

and $\xi_{1}^{T}, \Upsilon_{1}^{T}, \Sigma_{4}, \Sigma_{5}$, and $\Sigma_{6}$ are defined in (45).

It can be clearly shown that

$$
\begin{aligned}
\partial V(t) \triangleq & \sum_{i=1}^{r} \lambda_{i}^{2}(\theta(t)) \xi_{1}^{T} \Sigma_{3 i i} \xi_{1} \\
& +\sum_{i=1}^{r} \sum_{i<j}^{r} \lambda_{i}(\theta(t)) \lambda_{j}(\theta(t)) \xi_{1}^{T}\left(\Sigma_{3 i j}+\Sigma_{3 j i}\right) \xi_{1} \\
& +\sum_{i=1}^{n} \Upsilon_{1}^{T}(t) \Sigma_{4} \Upsilon_{1}(t)+\sum_{i=1}^{h_{1} / T} \Upsilon_{1}^{T}(t) \Sigma_{5} \Upsilon_{1}(t) \\
& +\sum_{i=n+1}^{h_{2} / T} \Upsilon_{1}^{T}(t) \Sigma_{6} \Upsilon_{1}(t)
\end{aligned}
$$

Premultiplying and postmultiplying $\Sigma_{3 i i}$ by $\operatorname{diag}\left\{P^{-1} P^{-1} P^{-1} P^{-1} P^{-1}\right\}, \quad$ premultiplying and postmultiplying $\Sigma_{4}, \Sigma_{5}, \Sigma_{6}$ by $\operatorname{diag}\left\{P^{-1} P^{-1} P^{-1}\right\}$, and letting $G=P^{-1}, \bar{R}_{1}=P^{-1} R_{1} P^{-1}, \bar{R}_{2}=P^{-1} R_{2} P^{-1}, \bar{Q}_{1}=P^{-1} Q_{1} P^{-1}$, $\bar{Q}_{2}=P^{-1} Q_{2} P^{-1}, \bar{Z}=P^{-1} Z P^{-1}, \bar{N}_{1}=P^{-1} N_{1} P^{-1}, \bar{N}_{2}=P^{-1} N_{2} P^{-1}$, $\bar{M}_{1}=P^{-1} M_{1} P^{-1}, \bar{M}_{2}=P^{-1} M_{2} P^{-1}, \bar{S}_{1}=P^{-1} S_{1} P^{-1}, \bar{S}_{2}=P^{-1} S_{2} P^{-1}$, $\bar{X}_{11}=P^{-1} \widetilde{X}_{11} P^{-1}, \bar{X}_{12}=P^{-1} \widetilde{X}_{12} P^{-1}, \bar{X}_{22}=P^{-1} \widetilde{X}_{22} P^{-1}$, $\bar{Y}_{11}=P^{-1} Y_{11} P^{-1}, \bar{Y}_{12}=P^{-1} Y_{12} P^{-1}, \bar{Y}_{22}=P^{-1} Y_{22} P^{-1}$, and $\widetilde{K}_{1 i}=K_{1 i} P^{-1}$ yield $\Psi_{4 i i}, \Psi_{1}, \Psi_{2}$, and $\Psi_{3}$. Following a similar line of the previous process to $\Sigma_{3 i j}$ and $\Sigma_{3 j i}$ yields $\Psi_{4 i j}$ and $\Psi_{4 j i}$.

Since $\Psi_{4 i i}<0$ holds for $1 \leq i \leq r$, and $\left(\Psi_{4 i j}+\Psi_{4 j i}\right)<0$ holds for $1 \leq i<j \leq r, \Sigma_{4}<0, \Sigma_{5}<0$, and $\Sigma_{6}<0$, then we have $\partial V(t)<0$. Therefore the fuzzy delta operator system (3)
TABLE 1: Comparisons of maximum allowed delay upper bound $h_{2}$ for Example 12 with $h_{1}=0.8$.

\begin{tabular}{lc}
\hline Method & $h_{2}(T=0.01)$ \\
\hline Result of Corollary 9 & Infeasible \\
Result of Theorem 8 & 0.933 \\
\hline
\end{tabular}

under the controller (59) is asymptotically stable. Finally, the explicit expression of the state feedback controller is given by $K_{1 i}=\widetilde{K}_{1 i} G^{-1}$. The proof is completed.

\section{Simulation Examples}

In this section, three examples are provided to demonstrate the effectiveness of the proposed results.

Example 12 (Stability Analysis). Consider a T-S fuzzy delta operator system with time-varying delay in the form of (1) with parameters given by

$$
\begin{array}{ll}
A_{1}=\left[\begin{array}{cc}
-2 & 0 \\
0 & -0.9
\end{array}\right], & A_{2}=\left[\begin{array}{cc}
-1 & 0.5 \\
0 & -1
\end{array}\right], \\
A_{d 1}=\left[\begin{array}{cc}
-1 & 0 \\
-1 & -1
\end{array}\right], & A_{d 2}=\left[\begin{array}{cc}
-1 & 0 \\
0.1 & -1
\end{array}\right] .
\end{array}
$$

In this example, for a given delay lower bound $h_{1}=0.8$, we seek for the admissible upper bound $h_{2}$, which guarantees the asymptotic stability of the open-loop system. Choosing the sampling period $T=0.01$, the obtained results are listed in Table 1.

Table 1 shows that the proposed result in Theorem 8 is less conservative than that in Corollary 9, which demonstrates the advantages of our method. Table 2 shows the delay upper bound $h_{2}$ under different delay lower bound $h_{1}$ and different sampling period $T$. It is obvious that the delay upper bound $h_{2}$ increases gradually as the sampling rate rises, which indicates the advantage of the delta operator fuzzy system at high sampling rate.

Example 13 (Controller Design). To further illustrate the effectiveness of our method for controller design, we consider the following T-S fuzzy delta operator system with timevarying delay:

$$
\partial V(t)=\sum_{i=1}^{r} \lambda_{i}(\theta(t))\left[A_{i} x(t)+A_{d i} x(t-n T)+B_{i} u(t)\right],
$$

where $A_{i}, B_{i}$, and $A_{d i}(i=1,2)$ are given by

$$
\begin{gathered}
A_{1}=\left[\begin{array}{cc}
0 & 0.6 \\
0 & 1
\end{array}\right], \quad A_{d 1}=\left[\begin{array}{cc}
0.5 & 0.9 \\
0 & 2
\end{array}\right], \\
B_{1}=\left[\begin{array}{l}
1 \\
1
\end{array}\right], \quad A_{2}=\left[\begin{array}{ll}
1 & 0 \\
1 & 0
\end{array}\right], \\
A_{d 2}=\left[\begin{array}{cc}
0.9 & 0 \\
1 & 1.6
\end{array}\right], \quad B_{2}=\left[\begin{array}{l}
1 \\
1
\end{array}\right] .
\end{gathered}
$$


TABLE 2: Comparisons of maximum allowed delay upper bound $h_{2}$ by different $h_{1}$ and $T$ for Example 12 .

\begin{tabular}{|c|c|c|c|c|c|c|c|c|c|}
\hline Method & & $T=0.0$ & & & $=0.0$ & & & $T=0.1$ & \\
\hline$h_{1}$ & 0.1 & 0.4 & 0.8 & 0.1 & 0.4 & 0.8 & 0.1 & 0.4 & 0.8 \\
\hline Result of Theorem 8 & 0.732 & 0.790 & 0.933 & 0.685 & 0.749 & 0.901 & 0.626 & 0.700 & 0.863 \\
\hline
\end{tabular}

TABLE 3: Comparisons of maximum allowed delay upper bound $h_{2}$ by different $h_{1}$ and $T$ for Example 13 .

\begin{tabular}{lccccccccc}
\hline Method & \multicolumn{3}{c}{$T=0.01$} & \multicolumn{3}{c}{$T=0.05$} & \multicolumn{3}{c}{$T=0.1$} \\
\hline$h_{1}$ & 0.1 & 0.4 & 0.8 & 0.1 & 0.4 & 0.8 & 0.1 & 0.4 & 0.8 \\
Result of Corollary 11 & 0.185 & - & - & 0.179 & - & - & 0.172 & - & - \\
Result of Theorem 10 & 0.428 & 0.668 & 0.951 & 0.418 & 0.658 & 0.941 & 0.406 & 0.646 & 0.929 \\
\hline
\end{tabular}

For different delay lower bounds $h_{1}$, the allowed delay upper bounds $h_{2}$ are listed in Table 3 . It can be seen that the proposed results in Theorem 10 are less conservative than those in Corollary 11.

The fuzzy controller gains for $T=0.01, h_{1}=0.8$, and $h_{2}=0.951$ by Theorem 10 are given as

$$
\begin{aligned}
K_{11} & =\left[\begin{array}{ll}
1.2781 & -4.4103
\end{array}\right], \\
K_{12} & =\left[\begin{array}{ll}
0.0812 & -2.9757
\end{array}\right], \\
K_{21} & =\left[\begin{array}{ll}
-0.1010 & -2.0993
\end{array}\right], \\
K_{22} & =\left[\begin{array}{ll}
-1.0592 & -2.5416
\end{array}\right], \\
K_{31} & =\left[\begin{array}{ll}
-0.1064 & -1.9295
\end{array}\right], \\
K_{32} & =\left[\begin{array}{ll}
-1.0592 & -2.5414
\end{array}\right] .
\end{aligned}
$$

Example 14. To illustrate the application of our method, we consider the following truck-trailer system given in [32]:

$$
\begin{gathered}
\dot{x}_{1}(t)=-c \frac{v t_{1}}{L t_{0}} x_{1}(t)-(1-c) \frac{v t_{1}}{L t_{0}} x_{1}(t-d(t))+\frac{v t_{1}}{l t_{0}} u(t), \\
\dot{x}_{2}(t)=c \frac{v t_{1}}{L t_{0}} x_{1}(t)+(1-c) \frac{v t_{1}}{L t_{0}} x_{1}(t-d(t)), \\
\dot{x}_{3}(t)=\frac{v t_{1}}{t_{0}} \sin x_{2}(t)+c \frac{v t_{1}}{2 L} x_{1}(t) \\
+(1-c) \frac{v t_{1}}{2 L} x_{1}(t-d(t))
\end{gathered}
$$

where $x_{1}(t)$ is the angular difference between the truck and trailer, $x_{2}(t)$ is the angle of the trailer, and $x_{3}(t)$ is the vertical position of rear end of the trailer.

The model parameters are given as $l=2.8, L=5.5$, $v=-1.0, t_{1}=2.0$, and $t_{0}=0.5$, and $c \in[0,1]$ is a retarded coefficient with limits 0 and 1 corresponding to delay-free term and to a full-delay term. The premise variable is chosen as $\theta(t)=x_{2}(t)+c\left(v t_{1} / L t_{0}\right) x_{1}(t)+(1-c)\left(v t_{1} / L t_{0}\right) x_{1}(t-d(t))$, and the sampling period $T=0.01$. The following fuzzy rules via delta operator are employed by

Plant Rule 1: IF $\theta(t)=$ is about $0 \mathrm{rad}$, THEN

$$
\partial x(t)=A_{1} x(t)+A_{d 1} x(t-n T)+B_{1} u(t),
$$

Plant Rule 2: $\operatorname{IF} \theta(t)=$ is about $\pi \mathrm{rad}$ or $-\pi \mathrm{rad}$, THEN

$$
\partial x(t)=A_{2} x(t)+A_{d 2} x(t-n T)+B_{2} u(t) .
$$
by

The membership functions for Rule 1 and Rule 2 are given

$$
\lambda=\left\{\begin{aligned}
\lambda_{1}= & \left(1-\frac{1}{1+\exp (-3(\theta(t)-0.5 \pi))}\right) \\
& \times\left(1-\frac{1}{1+\exp (-3(\theta(t)+0.5 \pi))}\right), \\
\lambda_{2}= & 1-\lambda_{1},
\end{aligned}\right.
$$

and with

$$
\begin{gathered}
A_{1}=\left[\begin{array}{ccc}
0.509 & 0 & 0 \\
-0.509 & 0 & 0 \\
0.509 & -4 & 0
\end{array}\right], \\
A_{d 1}=\left[\begin{array}{ccc}
0.218 & 0 & 0 \\
-0.218 & 0 & 0 \\
0.218 & 0 & 0
\end{array}\right], \\
B_{1}=\left[\begin{array}{c}
-1.4286 \\
0 \\
0
\end{array}\right],
\end{gathered}
$$

$$
\begin{gathered}
A_{2}=\left[\begin{array}{ccc}
0.509 & 0 & 0 \\
-0.509 & 0 & 0 \\
0.810 & -6.366 & 0
\end{array}\right], \\
A_{d 2}=\left[\begin{array}{ccc}
0.218 & 0 & 0 \\
-0.218 & 0 & 0 \\
0.347 & 0 & 0
\end{array}\right], \\
B_{2}=\left[\begin{array}{c}
-1.4286 \\
0 \\
0
\end{array}\right]
\end{gathered}
$$




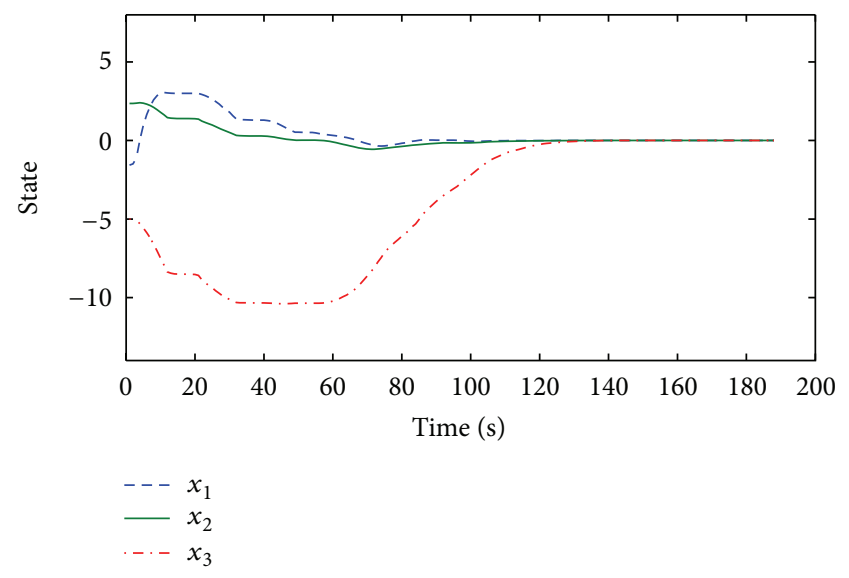

FIGURE 1: State responses for the closed-loop system in Example 14.

Assume the time-varying delay $1 \leq n T \leq 2$, and the initial condition $x\left(t_{0}\right)=\left[\begin{array}{ll}-0.5 \pi & 0.75 \pi-5\end{array}\right]^{T}$. The fuzzy delta operator controller gains by Theorem 10 are given as

$$
\begin{aligned}
& K_{11}=\left[\begin{array}{lll}
2.2108 & -2.7252 & 0.1445
\end{array}\right], \\
& K_{12}=\left[\begin{array}{lll}
2.2620 & -3.0776 & 0.1453
\end{array}\right], \\
& K_{21}=\left[\begin{array}{lll}
0.5423 & 0.0608 & -0.0046
\end{array}\right], \\
& K_{22}=\left[\begin{array}{lll}
0.5656 & 0.0503 & -0.0038
\end{array}\right], \\
& K_{31}=\left[\begin{array}{lll}
0.5467 & 0.0095 & -0.0015
\end{array}\right], \\
& K_{32}=\left[\begin{array}{lll}
0.5689 & 0.0079 & -0.0012
\end{array}\right]
\end{aligned}
$$

As shown in Figure 1, the states of the closed-loop system converge to zero under the obtained fuzzy delta operator state-feedback controller, which demonstrates the effectiveness of our method.

\section{Conclusion}

This paper proposes an input-output method to analysis and synthesis of T-S fuzzy delta operator systems with timevarying delay. The two-term approximation method has been employed to transform the fuzzy delta operator system with time-varying delay into a feedback interconnection form. Based on a Lyapunov-Krasovskii functional in delta operator domain, the SSG method is suggested for the interconnected system. Numerical examples are given to demonstrate the advantages and less-conservatism of the proposed results.

\section{Acknowledgments}

The work described in this paper was supported in part by the National Natural Science Foundation of China (nos. 61104112 and 61004038), in part by the Fundamental Research Funds for the Central Universities (no. HIT. NSRIF. 201162), and the China Postdoctoral Science Foundation (no. 2012M510960).

\section{References}

[1] M. Sugeno, Industrial Applications of Fuzzy Control, Elsevier, New York, NY, USA, 1985.

[2] T. Tanaka and H. O. Wang, Fuzzy Control Systems Design and Analysis: A Linear Matrix Inequality Approach, John Wiley \& Sons, New York, NY, USA, 2001.

[3] J. B. Qiu, G. Feng, and H. J. Gao, "Fuzzy-model-based piecewise $\mathscr{H}_{\infty}$ static-output-feedback controller design for networked nonlinear systems," IEEE Transactions on Fuzzy Systems, vol. 18, no. 5, pp. 919-934, 2010.

[4] J. B. Qiu, G. Feng, and H. J. Gao, "Nonsynchronized-state estimation of multichannel networked nonlinear systems with multiple packet dropouts via T-S fuzzy-affine dynamic models," IEEE Transactions on Fuzzy Systems, vol. 19, no. 1, pp. 75-90, 2011.

[5] S. G. Cao, N. W. Rees, and G. Feng, "Analysis and design for a class of complex control systems. II. Fuzzy controller design," Automatica, vol. 33, no. 6, pp. 1029-1039, 1997.

[6] M. Chadli and H. Karimi, "Robust observer design for unknown inputs Takagi-Sugeno models," IEEE Transactions on Fuzzy Systems, 2012.

[7] M. Chadli and T. M. Guerra, "LMI solution for robust static output feedback control of discrete Takagi-Sugeno fuzzy models," IEEE Transactions on Fuzzy Systems, vol. 20, no. 6, pp. 1160-1165, 2012.

[8] G. Feng, "A survey on analysis and design of model-based fuzzy control systems," IEEE Transactions on Fuzzy Systems, vol. 14, no. 5, pp. 676-697, 2006.

[9] K. Gu, V. Kharitonov, and J. Chen, Stability of Time-Delay Systems, Birkhauser, Boston, Mass, USA, 2003.

[10] S. Xu and J. Lam, "Robust $\mathscr{H}_{\infty}$ control for uncertain discretetime-delay fuzzy systems via output feedback," IEEE Transactions on Fuzzy Systems, vol. 13, no. 1, pp. 82-93, 2005.

[11] Z. Zuo and Y. Wang, "Robust stability and stabilisation for nonlinear uncertain time-delay systems via fuzzy control approach," IET Control Theory \& Applications, vol. 1, no. 1, pp. 422-429, 2007.

[12] H. N. Wu and H. X. Li, "New approach to delay-dependent stability analysis and stabilization for continuous-time fuzzy systems with time-varying delay," IEEE Transactions on Fuzzy Systems, vol. 15, no. 3, pp. 482-493, 2007.

[13] J. B. Qiu, G. Feng, and H. J. Gao, "Observer-based piecewise affine output feedback controller synthesis of continuous-time T-S fuzzy affine dynamic systems using quantized measurements," IEEE Transactions on Fuzzy Systems, vol. 20, no. 6, pp. 1046-1062, 2012.

[14] H. Y. Li, B. Chen, Q. Zhou, and W. Y. Qian, "Robust stability for uncertain delayed fuzzy hopfield neural networks with Markovian jumping parameters," IEEE Transactions on Systems, Man, and Cybernetics B, vol. 39, no. 1, pp. 94-102, 2009.

[15] H. Y. Li, H. H. Liu, H. J. Gao, and P. Shi, "Reliable fuzzy control for active suspension systems with actuator delay and fault," IEEE Transactions on Fuzzy Systems, vol. 20, no. 2, pp. 342-357, 2012.

[16] L. G. Wu and W. X. Zheng, "Weighted $\mathscr{H}_{\infty}$ model reduction for linear switched systems with time-varying delay," Automatica, vol. 45, no. 1, pp. 186-193, 2009.

[17] L. G. Wu, X. G. Su, and P. Shi, "Sliding mode control with bounded $\mathscr{L}_{2}$ gain performance of Markovian jump singular time-delay systems," Automatica, vol. 48, no. 8, pp. 1929-1933, 2012. 
[18] R. Middleton and G. Goodwin, "Improved finite word length characteristics in digital control using delta operators," IEEE Transactions on Automatic Control, vol. 31, no. 11, pp. 1015-1021, 1986.

[19] M. A. Garnero, G. Thomas, B. Caron, J. F. Bourgeois, and E. Irving, "Pseudocontinuous identification application to adaptive pole placement control using the delta-operator," RairoAutomatique-Productique Informatique Industrielle-Automatic Control Production Systems, vol. 26, no. 2, pp. 147-166, 1992.

[20] G. Li and M. Gevers, "Comparative study of finite wordlength effects in shift and delta operator parameterizations," IEEE Transactions on Automatic Control, vol. 38, no. 5, pp. 803-807, 1993.

[21] C. P. Neuman, "Transformations between delta and forward shift operator transfer-function models," IEEE Transactions on Systems Man and Cybernetics, vol. 23, no. 2, pp. 295-296, 1993.

[22] K. Premaratne, R. Salvi, N. R. Habib, and J. P. LeGall, "Delta-operator formulated discrete-time approximations of continuous-time systems," IEEE Transactions on Automatic Control, vol. 39, no. 3, pp. 581-585, 1994.

[23] S. Zhou and T. Li, "Robust stabilization for delayed discretetime fuzzy systems via basis-dependent Lyapunov-Krasovskii function," Fuzzy Sets and Systems, vol. 151, no. 1, pp. 139-153, 2005.

[24] J. Qiu, G. Feng, and J. Yang, "A new design of delay-dependent robust $\mathrm{H} 1$ filtering for discrete-time T-S fuzzy systems with time-varying delay," IEEE Transactions on Fuzzy Systems, vol. 17, no. 5, pp. 1044-1058, 2009.

[25] H. Gao and T. Chen, "Stabilization of nonlinear systems under variable sampling: a fuzzy control approach," IEEE Transactions on Fuzzy Systems, vol. 15, no. 5, pp. 972-983, 2007.

[26] Z. R. Xiang, Q. W. Chen, W. L. Hu, and D. J. Zhang, "Robust stability analysis and control for fuzzy systems with uncertainties using the delta operator," Control and Decision, vol. 18, no. 6, pp. 720-723, 2003.

[27] D. Q. Li, C. Y. Sun, and S. M. Fei, "Stabilizing controller synthesis of delta-operator formulated fuzzy dynamic systems," Proceedings of the International Conference on Machine Learning and Cybernetics, no. 1, pp. 417-422, 2004.

[28] H. Yang, P. Shi, J. Zhang, and J. Qiu, "Robust $\mathscr{H}_{\infty}$ control for a class of discrete time fuzzy systems via delta operator approach," Information Sciences, vol. 184, pp. 230-245, 2012.

[29] X. Jiang, Q. L. Han, and X. Yu, "Stability criteria for linear discrete-time systems with interval-like time-varying delay," in Proceedings of the American Control Conference, pp. 2817-2822, 2005.

[30] J. Qiu, Y. Xia, H. Yang, and J. Zhang, "Robust stabilisation for a class of discrete-time systems with time-varying delays via delta operators," IET Control Theory \& Applications, vol. 2, no. 1, pp. 87-93, 2008.

[31] K. Gu, Y. Zhang, and S. Xu, "Small gain problem in coupled differential-difference equations, time-varying delays, and direct Lyapunov method," International Journal of Robust and Nonlinear Control, vol. 21, no. 4, pp. 429-451, 2011.

[32] H. J. Gao, Z. D. Wang, and C. H. Wang, "Improved $\mathscr{H}_{\infty}$ control of discrete-time fuzzy systems: a cone complementarity linearization approach," Information Sciences, vol. 175, no. 1-2, pp. 57-77, 2005. 


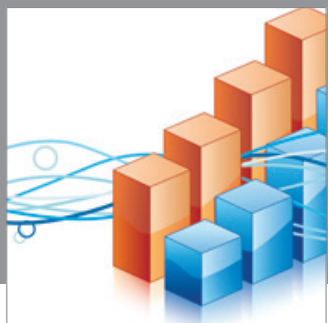

Advances in

Operations Research

mansans

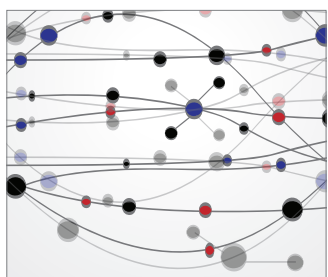

The Scientific World Journal
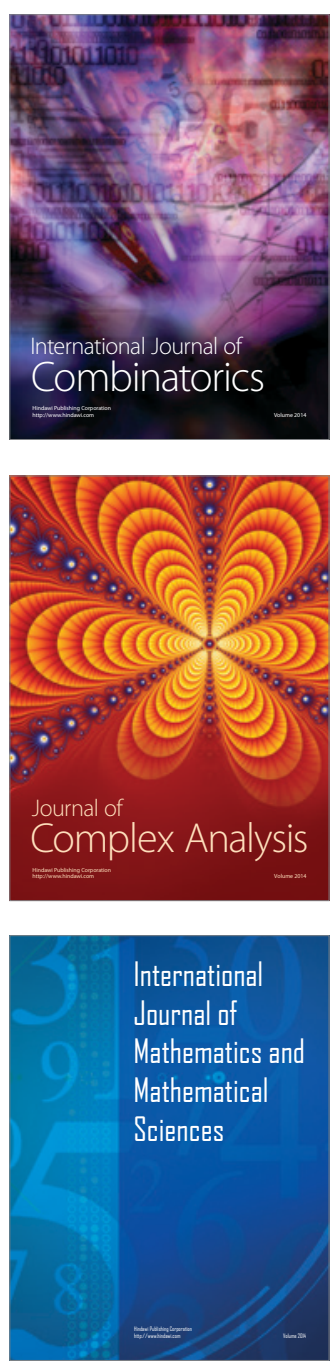
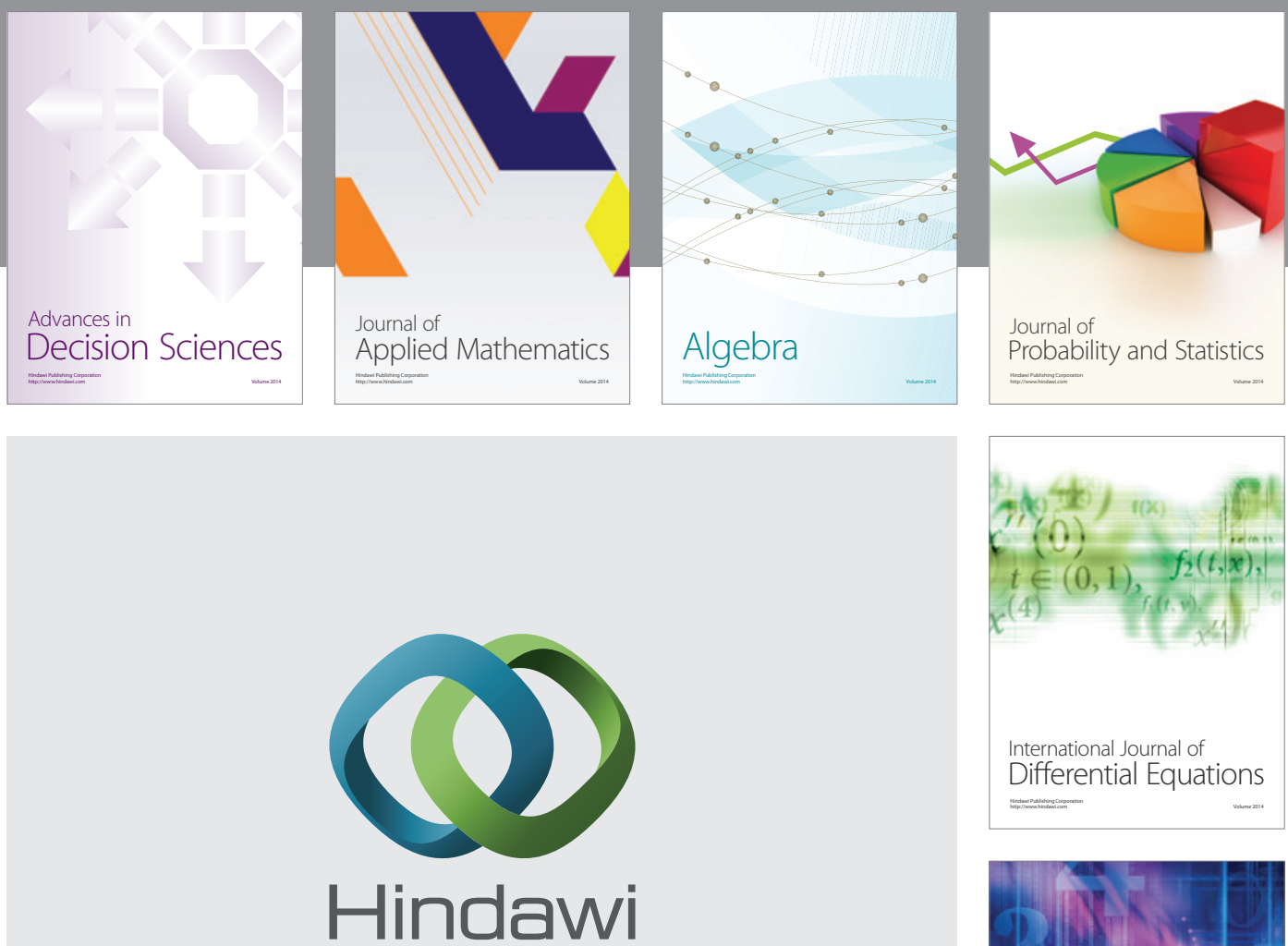

Submit your manuscripts at http://www.hindawi.com
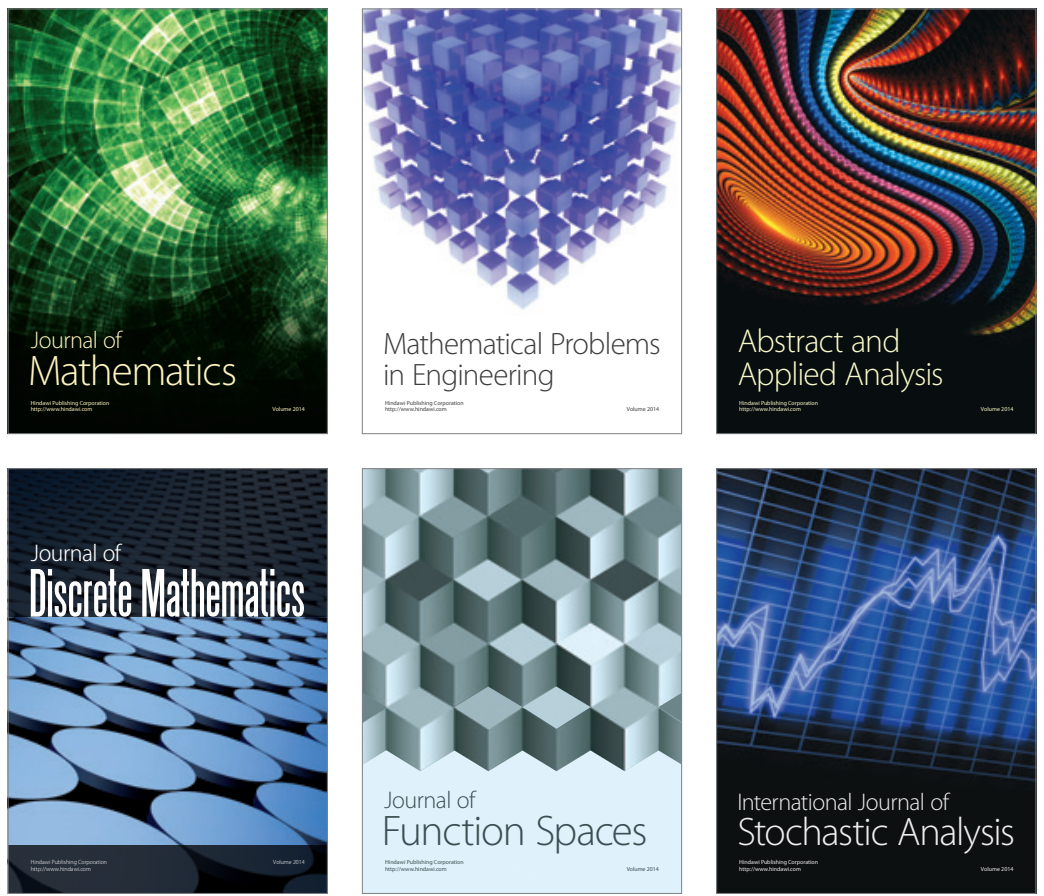

Journal of

Function Spaces

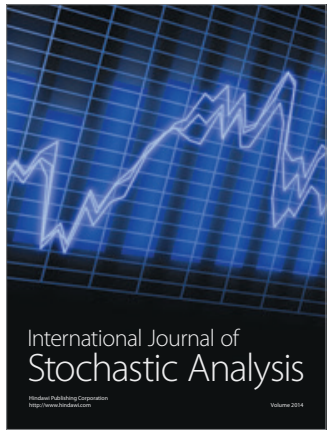

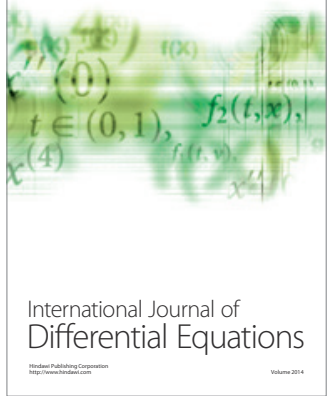
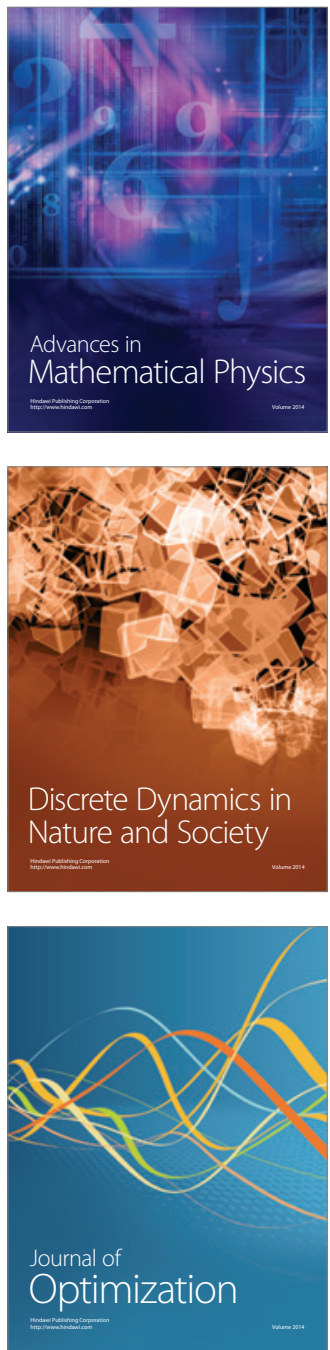